\title{
Interview with Eric Hoffert, Chairman of Versatility Software, Inc. and CEO of ShareMethods
}

\section{Eric Hoffert}

serves as Chairman of Versatility Software, Inc., a digital media and collaborative content consultancy and as CEO of ShareMethods, an online document sharing and collaboration service. Previously, he was CTO of Magnifi, a digital asset management provider; Multimedia Communications Manager at Apple Computer and a Research Scientist at AT\&T Bell Labs.

Keywords: Software-as-a-Service (SaaS), digital asset management service-oriented architecture (DAM SOA), rights-as-a-service, web services, social media, online office, Office 2.0, Web 2.0

\begin{abstract}
It's the end of content as we know it. Content creation, content management and content sharing have previously been separate worlds. Now they are colliding and converging into a single collaborative experience and platform. Where have we come from and where are we going? What can we deliver on today and what problems still need to be solved. What are the new opportunities? This interview addresses those key questions in a conversation between Michael Moon, Managing Editor of the DAM Journal, and Eric Hoffert, Chairman Versatility Software, and CEO, ShareMethods. Journal of Digital Asset Management (2007) 3, 288-296. doi:10.1057/palgrave.dam.3650105
\end{abstract}

MM: Eric - as a member of the editorial board for the Journal of DAM, you have had quite an opportunity to see the evolution of Digital Asset Management. Before we get into the particulars of your comments, let me just set up a framework for our conversation today.

Essentially, we'd like to address the notion of the end of content-as-we-know-it. And the notion that knowledge workers or media professionals create digital files.

In this new framework that we're going to be talking about with you - or this new framework that you'll be sharing with us, the notion that we're going to have "dumb files" with no knowledge in terms of what's in there - no profiling or no metadata around it, well that notion is basically disappearing, or in the process of disappearing. Especially in the context of Web 2.0 tools and things that we'll get into...

In our discussion today, we'll expand or perhaps revisit an expanded view or definition of digital assets. I'd like to at least stipulate that here for our conversation.

A digital asset consists of some sort of core media and/or data - described or profiled in some sort of set of metadata - or within a metadata scheme. Such that an outsider or someone not associated with creation of files knows the contents, and perhaps its status, in a work-in-process workflow. They may know the IP rights, clearances, rights and/or copyrights they're held in.

They may also have parameters or specifications for how to reuse or re-express this, vis-à-vis color space, fonts and other kinds of publishing parameters such as an online or highresolution print.

In that context, for the most part, I've talked in the Journal about media assets. And to a lesser extent, editorial assets - text. In some cases, we've talked about educational learning assets and knowledge assets - the products of knowledge workers.

We've talked about social assets. These are things that are attached to an interpersonal relationship — such as e-mail or discussion threads. Blogs. Wikis.

We also have software code assets. Those represent reusable software code. IT assets. In our framework today, we might characterize IT assets as IT service-management policies and configurations in metadata profiles for assets that are part of an IT infrastructure. That's a basic framework of various kinds of assets. 
I'd like you to speak to some of your perceptions or evolution of digital assets in that larger framework.

EH: Sure, Michael.

I think that if you go back historically, we certainly started with a lot of functionality in the DAM space that could be characterized as traditional enterprise software. People would install DAM systems behind a firewall and set up an isolated database of digital assets and content.

That approach would be used to manage content and assets that were produced by a different set of media software tools. Those tools typically would run on a desktop computer, and they would facilitate content creation.

Certainly, there was a lot of power for that traditional system in the time period in which it was developed. But the world is changing fairly dramatically, as you said. We are about to enter the period of the end-of-content-as-we-know-it. MM: This might be a good point to quickly define and/or differentiate content from an asset. EH: Okay. I'll let you take that one.

MM: As we started to create the conversation in the industry around DAM, we used to draw a pretty hard-line distinction. We said basically that digital assets represent digital files of some sort. Typically, media or editorial. Explicitly created for reuse and re-expression across multiple formats and media.

What makes a digital asset an asset? It entails the metadata describing the asset. And that the assets - that is to say the file and metadata live within a system with security policies and other sorts of policies for managing the appropriate access to, and use of, these assets.

In contrast, perhaps now a little more fuzzy but in the past, we used to say somewhat tongue-in-cheek - "Content is dumb." Wherein content represents a "dumb file." Where we know very little about what's inside.

Our ability to visualize - to develop a thumbnail or browser to it - was extremely limited. For the most part, content-management systems - were really about managing the flow of these files through a workflow.

Really, at the end of the day, most of the metadata associated with the content was more of a workflow management and/or status reporting about its position in an overall workin-process framework.

Yes.
MM: Those things began to merge, because a lot of the content creation tools and content management tools have evolved. Frankly, they kind of look a lot like DAM systems, today. EH: Yes.

MM: With that as a framework, you were going to say something about the evolution of content creation tools?

EH: Yes. If you again go back to those three primary points that I mentioned, DAM system as an enterprise software platform... the database of digital assets as a relatively isolated set of digital assets that are maintained and managed by that enterprise software. Then the desktop as the primary vehicle by which that set of digital assets is created.

I think if you look at all three of those core points from where DAM has come from - and you look at where we're going to - I think all three of those characteristics are about to go through a period of fairly dramatic change.

Certainly, DAM systems - many of them are starting to be provided as services. Where you have a DAM as a service. The databases of content being managed by these systems, and the intelligence of the schema behind those digital assets, is something where there's an increasing need to be able to share that database of digital assets across many systems as a service - of which the DAM system is only one facet in a much broader infrastructure.

Then a key trend that we're seeing is the increasing movement of content creation applications from the desktop to online. We've traditionally seen these desktop tools from Adobe, Microsoft and others. They're starting to be offered by a broader range of companies and are moving into the web browser with a whole new suite of collaboration capabilities.

Essentially, the notion of checking an asset in and out - which we've known for so long in a DAM system - is beginning to fall to the wayside as the asset and its creation service becomes an integral part of the system that's managing its metadata and allowing users to collaborate and so on.

I think all three of the core points of familiarity for the DAM community are all actually being challenged at their very core, as we move into a Web 2.0 framework. What used to be a balkanized and isolated database of content - essentially a Tower of Babel, where 
you couldn't speak the language from one DAM system to another... well, it's really coming to an end. It has to come to an end.

So the DAM systems themselves - are becoming more interoperable. It's easier to communicate now from one DAM system to another in terms of sharing content and sharing information, and sharing information between DAM systems and other systems - both in the enterprise and the consumer space. This is enabled by DAM-as-a-service, or Service Oriented Digital Asset Management.

This new world is blurring the lines between content creation and content management moving to one user experience that's more integrated, faster and more richly connected for collaboration. When you create an asset online now, its instantly available to your colleagues around the world.

As you pointed out, in terms of the social nature of these assets, there's a whole world exploding around the DAM community. For that though, we need to catch up - in terms of the social nature of assets, and the ability to find out what's the most popular and who's commenting on which assets. And the thread of discussions around assets, and the repurposing of those assets across both a business context and a consumer context. And having one framework for that. We aren't there yet and we need to leap forward.

The way that these trends are manifesting themselves in many ways is definitely as services. I think there's a whole world evolving around service-oriented DAM. Certainly some of it's taking place at an enterprise level. But quite a bit of it is being influenced by the consumer web.

MM: This actually might be a good place just to do a quick reprise of the four major waves of DAM.

EH: Yes.

MM: In the early- to mid-90s, basically, we had multimedia databases. Fetch and Canto, I think, were some of the original ones. They kind of existed on your desktop, and you used them as a personal productivity tool.

EH: Yes.

MM: Then from the mid-90s forward, the Web began to show up. We started getting grouped into departmental DAMs. One of the great early ones was Webware Mambo and MediaBank, and other ones emerged as really strong workgroup and departmental DAMs. It tended to be a 2-tier or 3-tier client server system. Where you had a dedicated client on the front end.

Then in the late-90s, and through the early 2000 s or maybe 2004 or 2005 , we had the real emergence of the enterprise DAMs and media service platforms. Those were full-tier web service-enabled DAMs. They pretty much assumed that the primary users of these solutions had browsers and big pipes and stable platforms and things like that.

This is where the whole notion of distributing media services through an enterprise - and maybe among some partners - really began to take hold. It was at that point that the marketing functions of large enterprises really started seeing a lot of utility. Whereas the media, entertainment and publishing - because they were kind of content factories, anyway perhaps saw it earlier.

Then around the 2005-2006 time-frame, we saw the emergence of what we called digital supply chains for content. "Supply chains" kind of implied two or three core ideas. One was end-to-end process integration. But more specifically, process integration of loosely coupled systems.

So you had multiple systems often times owned by very different people. But they had been wired or integrated in such a way that they could pass data and assets in a very controlled way. Hence the term, "Loosely coupled."

The other aspect of a supply chain is that it's an economic framework. And more specifically, a procurement framework, for bringing strategic sourcing and competitive bidding and vendor consolidation into a supply network. Thereby integrating these suppliers to your supply chain. They kind of begin to integrate their core processes with your core processes.

Now we're talking about something that's the next wave of innovation within this notion of network supply chains. You're beginning to describe the emergence of these distributed services within these digital supply chains. Can you speak a little more to that?

EH: Yes. Absolutely.

One of the things to look at in addition to the emergence of DAM as a service would be some of the higher-level constructs. You could 
consider process or workflow as a service. Behind that, there is the capability of function as a service.

When you have a greater capability to interconnect different databases of content, and mash up the applications that can operate on those databases of digital assets, you have a more fluid capability to invite employees, partners and suppliers to more readily collaborate with you around the content that you're working with.

There are some interesting developments in the industry that are happening in order to facilitate these capabilities.

Before I go through that, let's just take a step back and identify a few of the problems that I think hinder the progress that we could be making.

One of them is, it could be difficult to $\log$ in to a lot of different Web 2.0 or DAM 2.0, Office 2.0 applications for end-users.

MM: Eric, one of the things you might want to do here is just spend a paragraph or two on Web 2.0 as a concept. And link in the term of, "mash-up," and things like that.

EH: Sure. The definition of the term, "mashup." I talked briefly about the historical basis of enterprise DAM as a traditional piece of enterprise software, with an isolated database. And disconnected desktop tools to create and manage content.

It's been stated that a mash-up is a combination of applications that can be done in under a day. It's a convenient definition. As many people know, on the enterprise architecture side, an enterprise integration is a combination of applications where there's a need to share data or functionality across applications for end-users that typically takes far more than a day.

Actually, many enterprise integration projects can take certainly far more than just days. More typically - months, weeks, and in some cases, years.

What's happening for the Web 2.0 space is what happened in the consumer space in terms of consumer mash-ups. More recently with tools like PopFly from Microsoft and Google's mashup tools, we're starting to move into the small business area. And with applications like IBM's QED Wiki, we're moving into the enterprise space. We have the ability to combine application services from different vendors far more rapidly - more easily and more costeffectively than we ever could do with enterprise applications.

That is one of the hallmarks of Web 2.0. The ability to more easily combine applications. When you can do that, you can start to move away from the more traditionally isolated DAM system that has sat behind the firewall, into a DAM system that's more vibrant. It's more interconnected and has a greater level of readiness to support some of the goals behind social media.

As a sign of that, I have an involvement in an industry consortium called OpenSAM (www. opensam.org). OpenSAM stands for Open Simple Application Mash-up, and it was co-founded by the CEOs of iNetOffice and ShareMethods. One of its core goals is to facilitate a much easier interconnection of applications across the Internet, Intranets and Extranets (Figure 1).

You have a single login across multiple applications. You can more easily share traditional Office documents and digital assets across multiple applications. You can have common user-interface standards for ease of interacting with different applications from different providers.

There are many other areas that we'll be tackling in terms of standardization in what's called the Web 2.0, and also the Office 2.0 space that relate back into this.

But I do think it's extremely important. As I said before, we have some challenges right now. Many applications cannot talk to one another and are difficult to interconnect.

We're trying to tackle those problems headon with this industry consortium called

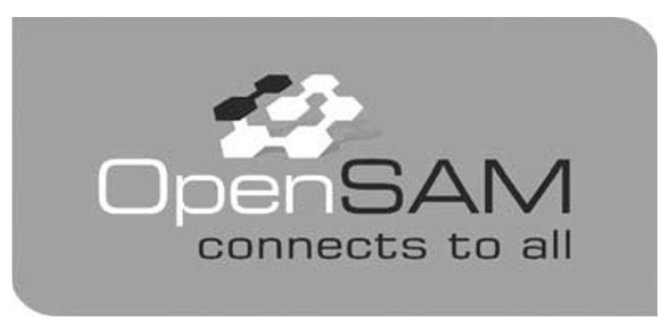

Figure 1: OpenSAM Badge

Open Simple Application Mashup "Connects to All" Badge highlights the key objective of connecting any application to any application, connecting any application to any content, and connecting people to applications (and by implication for social media, connecting people to people) (Credit: Tom Snyder, CEO, iNetOffice). 
OpenSAM. I think there are obviously other developments in the industry, as well, that are looking to do this. It's important to bring these developments back to the DAM community so that you can more readily realize this vision of DAM as a service.

To make it come to fruition, it doesn't mean wrapping a web-browser interface around the traditional DAM experience. It really has to address a number of key topics that we've been covering in our conversations.

The movement of content creation tools into the browser world, and integrating that with the management of assets. The interconnection of your digital assets into a social media framework. And the ability to more easily mash up applications and combine them, so that the digital assets that you're creating can be syndicated for revenue-generation. So that assets can flow into the digital supply chain that you've been talking about. And to do this in a way that's more fluid and more flexible than anything we've seen before in DAM (Figure 2).
That, I think, will bring us to the world of the end-of-content-as-we-know-it.

MM: This would be a great place to reprise you of an interview that I saw with Eric Schmidt on the Charlie Rose Show. It took place, I think, in April or so of 2006.

Charlie Rose asked Eric - the CEO of Google - "What's next? What's the next big wave of innovation?"

Eric said, "Well, you know, Charlie — in order to really answer that, we have to quickly reference what's been. Because largely, 'what's next...' kind of what we've been talking about — is a function of what's been." He said, "In one perspective, the 1980s were all about hardware. Hardware innovation. Out of that we got Seagate and Maxtor and EMC and Compaq and HP and Dell and Apple and IBM. The PC and the server."

Then he said, "By the time we got to the 90 s, there were no more big breakouts, in terms of hardware." Of course, they weren't really talking about the mobile space. That really wasn't a Silicon Valley thing.

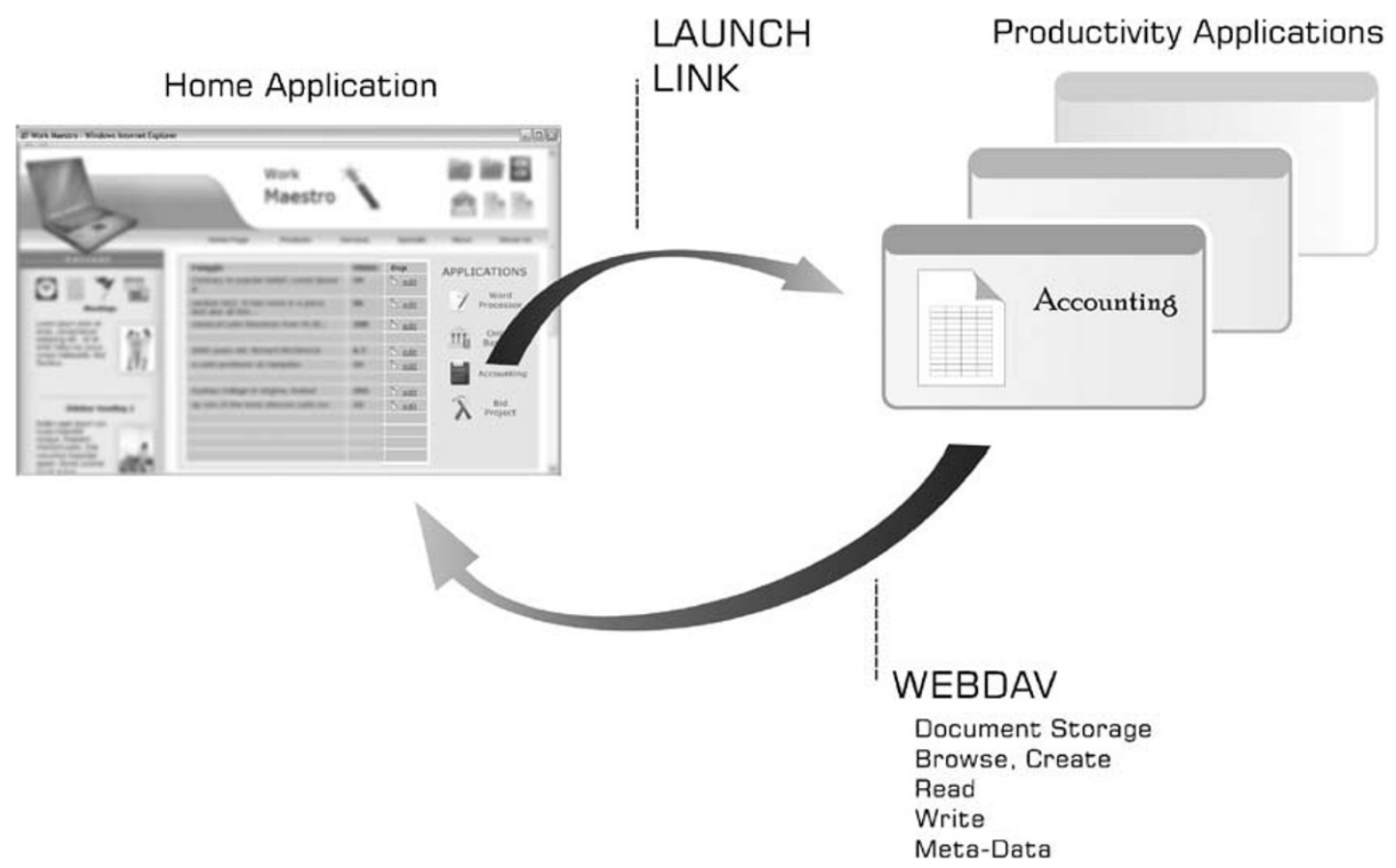

Figure 2: Open simple application mashup

Open Simple Application Mashup (OpenSAM) is shown with a home application (ie digital asset management service) launching multiple productivity applications (ie online image editor, online video editor, online word processor, online finance/rights management service). Each application can be a separate web service provided by a different software provider and linked together through the open standards of OpenSAM (Credit:Tom Snyder, CEO, iNetOffice). 
That aside, the 90s were all about enterprise software. Big software. Desktop software. The 90s was the decade that Microsoft really grew into its dominant position. Along with that, we got SAP, Oracle, Peoplesoft, Siebel, Computer Associates and so on.

Then he said, "The first six years of the 2000s were really about internet infrastructure," or what he called IP infrastructure. Out of that we got Google and Yahoo and eBay and Amazon. Those big Internet service platforms.

He said, "You know, as of 2006, that innovation space has all been used up." He said, "I can't tell you, Charlie, with the same 20/20 hindsight of what's been. But we're pretty certain that there are two facets, as far as the future."

On the basis of this, we will see all kinds of new innovative business models, and various and the next wave of wealth-creation.

He said, "The next wave is really about two things. Small groups of people that produce and consume small chunks of information and content." They produce and consume information - which you and I would call "user-generated content."

At some point - after so many turns of prosumption content - it hits critical mass and explodes into things like a Skype or Facebook or MySpace and other sorts of social media. Socialized what I will now call "Socialized Assets."

By the way, Eric and Google have already written close to $\$ 4$ billion of checks against that idea. When you look at all their acquisitions lately, they almost always entail these social networks or networks of socialized assets.

So the idea is that user-generated content and the larger framework of social collaboration associated with content creation - is essential and ground zero of this next wave of wealthcreation.

I think that seems to be a great lead-in to what you were beginning to talk about in terms of functions as a service. And how does that affect things like word processing, documents, Excel Spreadsheets, presentations, photo image manipulation and so on?

$\mathbf{E H}$ : Yes. I think the trend there... Of course we're seeing it from Google, with Google Apps and Google Docs and Google
Spreadsheets and Google Presentations. And there are other key players - ThinkFree, Zoho, ShareOffice, etc.

We just saw an announcement today from one of the flagship providers of desktop software - Adobe. They bought a company called "Virtual Ubiquity," which has an online word processor that's Flash-based now called Buzzword. And Adobe announced a service called "Share," for online document sharing.

The companies that have traditionally brought us the desktop tools, where you work on your creative content in isolation... They're starting to make the significant shift to moving that whole process online to the Internet, and - by implication - make the process of creating the content a more collaborative, social process. Adobe recently stated that flavors of Photoshop will be moving online too...

Online word processors from companies such as iNetWord and others - these are tools that we're starting to see come online which are initially happening for the Online Office space... Next we will see trends for the tools to create assets near and dear to the hearts of DAM practitioners, like PhotoShop and iMovie for example moving online.

The whole process, historically, has been a single-user or small workgroup. In isolation, usually - in a local work mode. I think that that whole model is going to change dramatically.

Not only will it change dramatically from the professional content creators, but I think tying back to Eric Schmidt's comments from Google - there's an explosion, of course, in content creation.

We've got companies like Yahoo acquiring JumpCut - where people can do online remixing of video with a budget of zero dollars. The accessibility of the tools for content creation, editing and remixing is also being transformed.

You see the work process and the workflow itself is moving online. Becoming more collaborative for the professional content creators. It's becoming accessible from a usergenerated content standpoint, in a way that it's never been before, for end-users. They'd typically just watch content passively, but they're now participating in its creation. 
Then you've got the intersection of these two worlds. Most major media companies now, when they're planning their presence on the Internet - they no longer think of it as a place where they publish their created digital assets that they worked on, and their traditional enterprise-class DAM systems.

Instead, they're thinking of it in a dual mode. They're thinking of it as a world in which the professional content creators who are building their editorial content can publish their media. That's a meeting place where the end-users the consumers of their publications - are posting their own content.

These two types of assets are coexisting now on the Internet in a bigger and bigger way. Obviously, the attention is focused largely on YouTube - where a lot of user content is produced and put online. But the reality is, a lot of the traditional media companies are also transforming their own websites and their own online presence, to incorporate this mixture of user-generated content and professionally produced content.

That's being enabled by a number of technical changes, which you mentioned. This notion of processes as a service, function as a service... We talked earlier about DAM as a service. I like to use the terms, "documents as a service." Or "content as a service."

All of these traditional desktop software, enterprise software functions are all being radically deconstructed and reassembled in a new way on the internet, where the internet is the application.

Just to give you some nice ways to think about this... I mentioned previously the industry consortium called OpenSAM. Open Simple Application Mash-up. The core tenets of OpenSam - there are three parts, and it starts with a universal statement. "Connects to all."

The idea behind OpenSAM is that any application should be able to connect to any other application. Number 1.

Number 2 - any application should be able to easily connect to any collection of content.

Number 3 - individuals or people should be able to more easily connect to applications.

So the simple phrasing of that is apps-to-apps, apps-to-content and people-to-apps. Well, guess what? What does that enable? What are we trying to do, at the end of the day with those three enablers, which we've targeted in this consortium called OpenSAM? It's about peopleto-people. At the end of the day, that's what we're seeking to enable.

We're seeking to enable a flexible Internet infrastructure that allows you to connect applications more easily together, hook up people to those applications, and allow applications to share content.

But at the end of the day, it's all about making connections from one person to another - one person to many other people - or from many people to many other people, using the Internet as application infrastructure.

I think that's where we're headed. I think that's a very fundamental change from historic DAM systems. I think also it's emblematic of the end-of-content-as-we-know-it.

MM: Picking up on that theme, the notion of end-of-content-as-we-know-it - one aspect of it means that no matter where I am in the world... office, home or maybe even mobile... I have access to a suite of tools. These tools may come from multiple vendors, or may be provisioned from multiple infrastructures.

But because I'm now a wired citizen, I have access to a burgeoning suite of tools that are interoperable. They share not only an application framework, but also share access to my "stuff." My content or my work files and so on.

The things to which I have access aren't just dumb files in a file repository. But they're brightly lit digital assets, with some pretty good metadata that's already built around it as a function of having used these networked tools.

As a function of that, what we used to call "content..." These kind of dumb, poorly lit, difficult-to-find, difficult-to-understand files, become brightly lit, pretty well profiled, and shareable and you might even say "promiscuous," files. I can wrap them or define them in certain rights and permissions policies. I can share them with people on an as-needed or need-to-know basis.

EH: That's very well said. I'd like to dig into a few of the things you said. Go a little deeper, to articulate them.

One of the biggest gaps that's facing us in this new world of digital assets as a service... It's absolutely essential for the DAM community to address, as a collection of companies and 
individuals... To have that really well-lit digital asset.

For years, we've talked about having robust metadata schemas associated with digital assets. I've often talked about the idea of those schemas traveling along with the assets. If not integral and embedded in the asset - like Adobe XMP, then at least a companion set of XML that's always traveling with the file as it moves from one system to another.

That's obviously very important in the world you've described. Where you can get to your content across any application - any device - from home, from work and so on.

A critical thing... and we've seen the need for this in the marketplace... Is those assets do need to be accompanied with two other essential pieces of information. One is a unique digital fingerprint that allows unambiguous identification of the assets. The other is business rules that are associated with that fingerprint and that asset. To define if it's a Creative Commons piece of content, or if it's something that requires compensation whenever it's reused. And if so, how much and to whom?

The rights management portion of the vision that we're describing is a gap, and a significant one. It's got to be closed. I don't think we'll truly realize the world of digital assets as a service until we've addressed that particular point.

Metadata is a powerful companion to a digital fingerprint, but as we all know, it's insufficient on its own. Especially for rights-holders and those who seek to be compensated for the use of their assets, the fingerprints and the business rules around royalties will be essential.

So as we move into this new world that we've talked about, with the applications for content creation moving online, where you can more flexibly build mash-ups across the applications... to build composite applications - leveraging the power of DAM... There is this other piece that has to be put into place on the rights management and royalty tracking and distribution side. That's essential to truly realize this vision that we're describing.

MM: It would sound to me that you're talking about a new business opportunity.

EH: I think it is. Yes.

MM: Rights as a service.
EH: Yes. That's very well said, Michael. I would say its rights as a service. Absolutely. I think it's one of the pieces that we need as a foundation in place before we can deliver on the bigger vision here.

When somebody wakes up, the way they'll think about it is, "What do I need to get done." They will basically be able to pick-and-choose the applications that they need. The apps would be dynamically provisioned, in a mash-up context. Where you would pay by the usage of each application as a subscription, and ensure that providers would be compensated accordingly for their application functionality. And likewise, in addition to picking and choosing the applications that you want, you should be able to pick and choose the content that you need.

That content might be Creative Commons and available to you for free within the appropriate Creative Commons places. Or it may be content that requires a set of business rules for royalty payments. And you should be able to - just like you as a consumer can buy a song that you like, and listen to it on your iPhone or your iPod for $\$ 0.99 \ldots$ You should be able to legitimately acquire the rights to use professional content, and be able to pay for it by the usage.

In the old days, people bought these stock photo CDs and paid $\$ 100,200$ or 300 and got rights to a variety of photographs - most of which they didn't want.

We need to be in a mode where you can buy just that one photograph that you want, and use it in the context that you need it, and do it in a way that is flexible. With that piece of content that you've embedded into your online presentation that you now share with others - if they want to use that same image - they should have an easier and more fluid and flexible way to use that image, and also have compensation paid to the rights holder.

The whole infrastructure that we have today needs to be restructured, to make it easier essentially, to have application mash-ups and content mash-ups. That's also very critical. Rights as a service - as you've very nicely identified it - would be a very important part of that (Figure 3). 


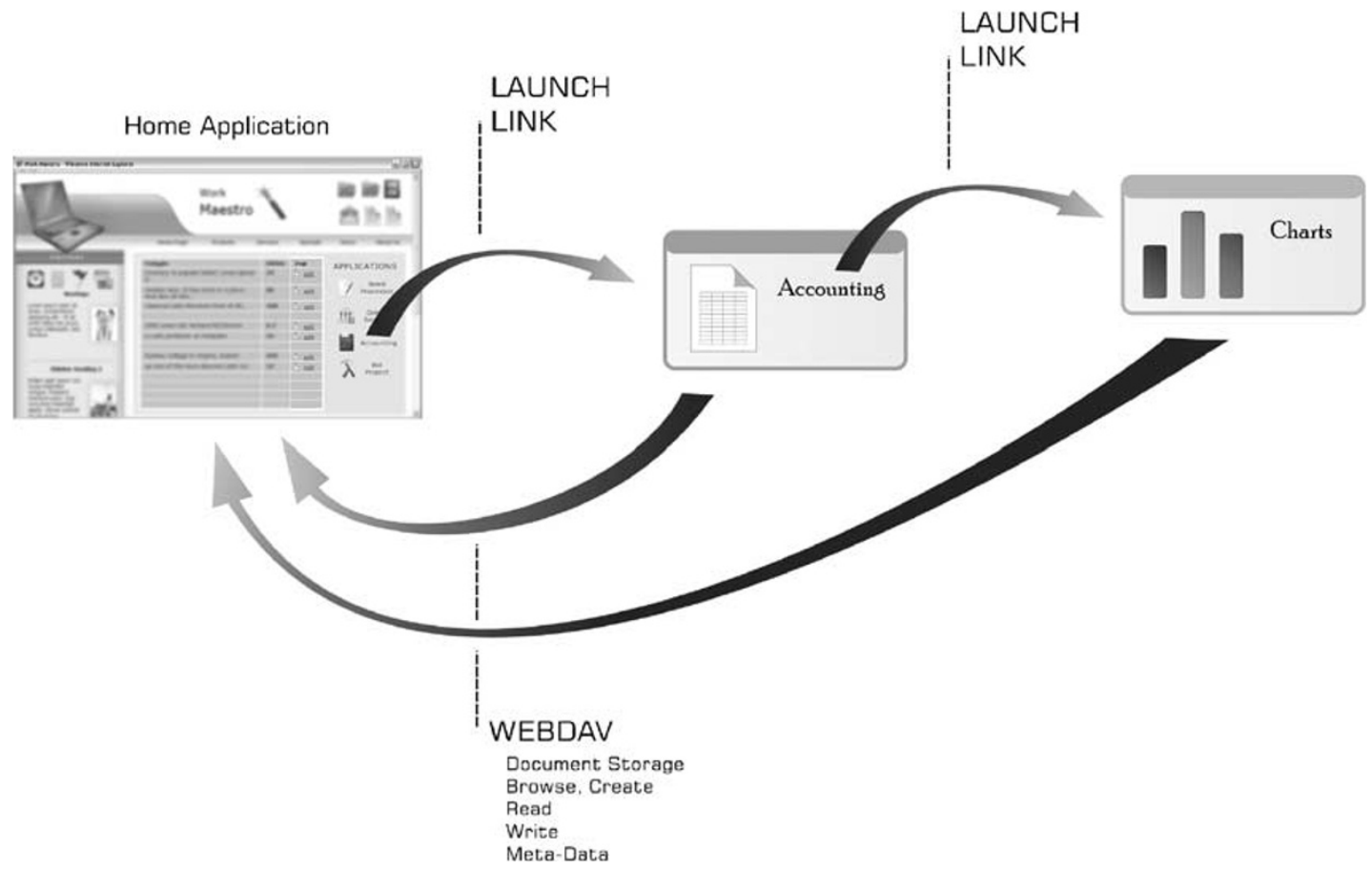

Figure 3: OpenSAM, shown with a CMS

Open Simple Application Mashup (OpenSAM) is shown with a home application (ie content management service) launching a productivity application (ie online spreadsheet), which is launching another productivity application (ie graphical charting or visualization service). The mash-up is generated dynamically with single sign-on, cross-application document sharing, and dynamic application provisioning (Credit:Tom Snyder, CEO, iNetOffice).

MM: Cool.

I think that's about it. Any last words, in terms of either the end-of-content-as-we-knowit, or the emerging social collaboration networks around the creation and management of assets? EH: This is all highly empowering to the enduser. When you are at work you'll now be able to more easily share, post, tag and comment on assets that are valuable to you - within your company. Either Office documents or digital media assets.

Likewise, the empowerment you get as an end-user, to create your own content, post it out on the consumer internet and share it with others... I think all of this is leading towards the greater empowerment of users - both in the corporate world and in the consumer world.

The face of content-as-we-know-it is changing, too. I think it's a really exciting time to be involved in the development of these tools, as well as to be an end-user, and get the benefit of using these new services.

MM: Well again, Eric - I want to thank you again for both participating as a member of our editorial board, and our really illuminating conversation today.

EH: Well, thank you. I enjoyed it very much, Michael.

MM: Okay. Be good. 